Eos, Vol. 88, No. 42, 16 October 2007

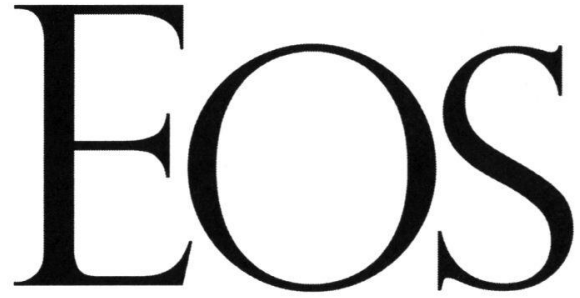

EOS, TRANSACTIONS, AMERICAN GEOPHYSICAL UNION
VOLUME 88 NUMBER 42

\section{OCTOBER 2007}

PAGES 425-440

\title{
Unraveling the Causes of Radiation Belt Enhancements
}

\section{PAGES $425-426$}

As technology advances, as society in general and scientists in particular become increasingly reliant on the artificial satellites that circle our planet, and as space agencies worldwide are considering new ways to send humans to the Moon and Mars, better understanding of adverse space weather in the Earth's upper atmosphere is crucial.

Critical to the success of satellite and manned spaceflight missions will be their ability to pass safely through Earth's Van Allen radiation belts. Energetic charged particles trapped in the radiation belts are a major source of damaging space weather effects not only on spacecraft and astronauts but also on life and society here on Earth, as such enhancements can create false signals in electronics and outages in vital satellite systems. Thus, understanding the physical processes that enhance the electron and ion content of our radiation belts is a timely and pressing issue.

However, scientists studying the radiation belts are hindered by the complexities of plasma flux through the belts-everything seems to happen at once, with competing source and loss processes driven by the same external factors. In addition, the fast speed of these particles makes the problem global in nature instead of localized to an easily studied area.

To shed light on these complex dynamics, we briefly describe mechanisms of plasma flux through the radiation belts, first for ions and then for electrons, and then discuss how these source and loss processes fit together to define the radiation belt response to geomagnetic disturbances.

\section{Radiation Belt Plasma}

The Earth's radiation belts are two concentric doughnuts of plasma that encircle our planet. As shown schematically in Figure 1, they tend to fill in the inner magnetosphere. There, the magnetic field generally

BY M.W. LIEMOHN AND A. A. CHAN reflects that of a classic dipole. The inner torus covers an altitude between 700 and 10,000 kilometers above the Earth, while the outer torus is larger, extending about 13,000-65,000 kilometers above the Earth. Both contain particles ionized by cosmic rays and solar flares, with the latter sometimes causing considerable changes in radiation belt particle flux as a result of the flares inducing intense geomagnetic storms.

While the radiation belts exist at the highest energies of the spectrum (the megaelectron volt range), the plasmasphere and ring current are lower-energy charged particle populations coexisting in the inner magnetosphere, in the electron volt and kiloelectron volt ranges, respectively. The plasmasphere dominates the mass content of the inner magnetosphere and the ring current dominates the energy content of this region, and therefore both play a special role in governing radiation belt physics.
Radiation belt particles move quickly. Electrons in the outer zone, for instance, circle around the belt's local magnetic field in a few milliseconds. They also bounce back and forth as they become trapped along the belt's magnetic field lines with a period of a few seconds or less. Finally, they drift across the local magnetic field direction in an eastward flow around the Earth, making a complete drift orbit in a few to a few tens of minutes. These three motions can be quantified in terms of energy-conserving "adiabatic" invariants [e.g., Roederer, 1970].

A magnetic field change that is slow compared with the drift motion yields a reversible increase or decrease in particle energy, following the adiabatic invariant relationships. During geomagnetic storms, the inner magnetospheric field inflates, and the drift paths correspondingly expand outward. This influence is known as the "Dst effect" [e.g., Kim and Chan, 1997], so named because the field inflation tracks the disturbance stormtime index, Dst. This process causes the radiation belt fluxes to undergo a predictable and repeatable decrease and subsequent increase during geomagnetic storms.

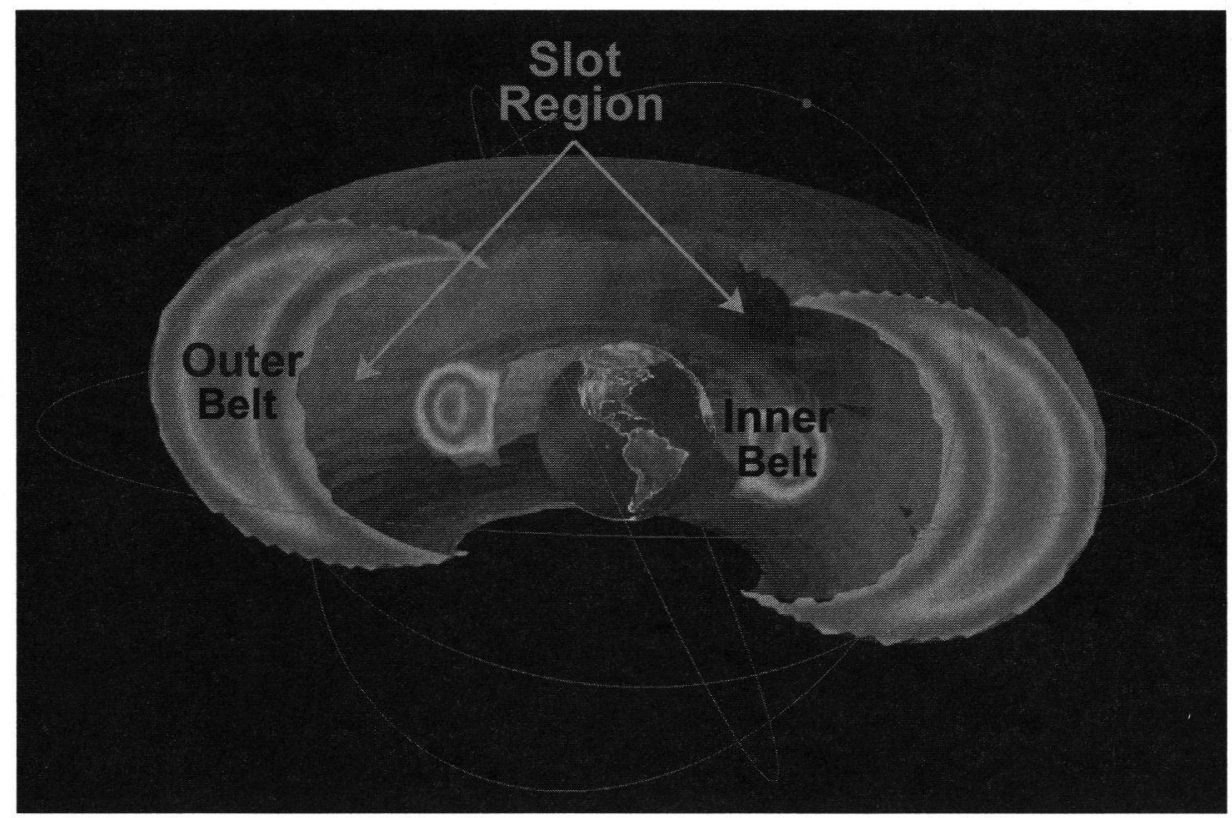

Fig. 1. Energetic electron flux, showing the inner and outer radiation belts. Warm colors indicate high flux intensity, while cool colors indicate low flux intensity values. Also shown are the orbits of three Global Positioning System (GPS) spacecraft and one geosynchronous spacecraft (the equatorial orbit). Image from AF-GEOSpace. Original color image appears at the back of this volume. 


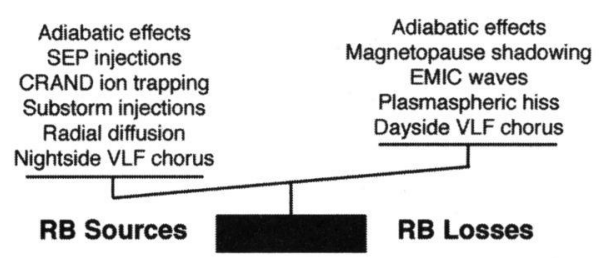

Fig. 2. Scale weighing the plasma source and loss mechanisms within the radiation belts. In this example, the scale is tipped, indicating a scenario where plasma is enhanced within the radiation belts. SEP stands for solar energetic particle; CRAND is cosmic ray albedo neutron decay; EMIC is electromagnetic ion cyclotron; and VLF is very low frequency.

This is not the only process at work, however. Throughout a geomagnetic storm, several source terms combine to enhance the radiation belts, while a variety of loss mechanisms simultaneously compete to suppress the radiation belt intensity. This balance between sources and losses is schematically shown in Figure 2. The balance is not always equal, and Reeves [1998] showed that roughly half of geomagnetic storms result in a flux increase, and the other half are about evenly split between a flux decrease and roughly no change from the prestorm flux intensity.

Some of these source and loss terms listed in Figure 2 are due to large-scale reconfigurations of the magnetosphere, while others are due to the interaction of the particles with plasma waves (oscillations in the magnetospheric electric and magnetic fields). In addition, the radiation belts include energetic ions as well as electrons, and some of these processes listed in Figure 2 are important for one or the other type of particle.

\section{Large-Scale and Global Processes}

An important source is when a galactic cosmic ray strikes the upper atmosphere, creates a neutron, and this neutron decays into a proton before it leaves the magnetosphere. This source, known as cosmic ray albedo neutron decay (CRAND), provides a small but continuous addition to the inner zone ions. An important but sporadic source mechanism of radiation belt ions is the trapping of solar energetic particles from solar flares. After these ions hit the magnetosphere, they circle in a wide orbit due to the presence of the local magnetic field. This large gyroradius allows some of the ions to cross into the radiation belts. This process often results in the sudden enhancement of the ion content within radiation belts

Additionally, radiation belt ions can escape through the boundary between the magnetosphere and interplanetary space (the magnetopause). As the drift paths expand with the inflated magnetic field, they can sometimes encounter the magnetopause. As a result, the adiabatic, reversible flux decrease of the Dst effect mani-

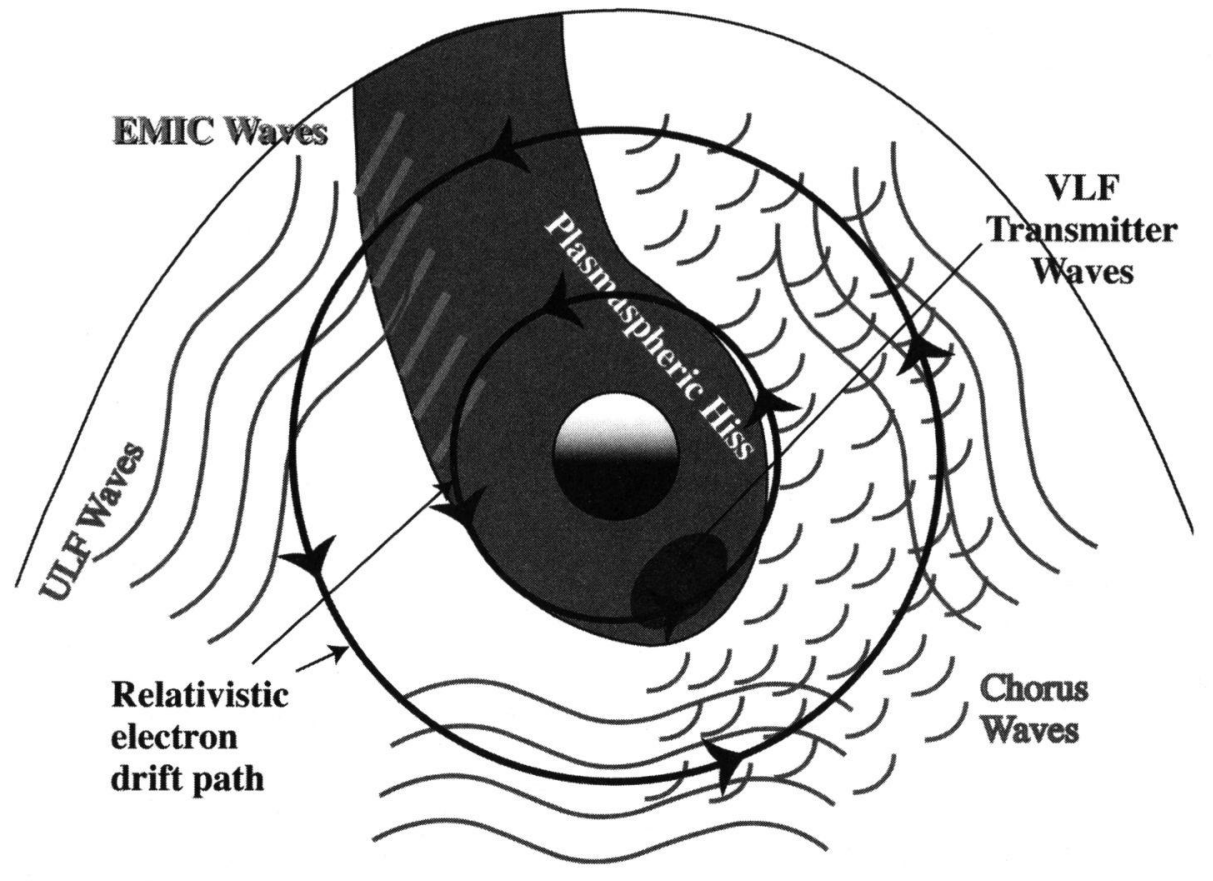

Fig. 3. A schematic of how relativistic electron paths pass through various plasma wave regions over the course of a drift period. The inner drift path will experience mostly loss processes, while the outer drift path encounters a mixture of acceleration and loss processes. Adapted from Summers et al. [1998]. Original color image appears at the back of this volume.

fests as real loss of particles from the radiation belts. Radiation belt ions may also be lost if their bounce path is altered enough so that they encounter the relatively dense upper atmosphere. However, this scattering process is relatively slow compared with the potentially rapid loss caused by encounters with the magnetopause.

The radiation belt electrons are also influenced by the large-scale magnetospheric structure. Similar to energetic ions, the electrons are also subject to the Dst effect and losses at the magnetopause. Another large-scale field effect, involving intense and sudden injections from the plasma sheet, results in an electron source. This process brings energetic particles from the magnetospheric tail deep into the inner magnetosphere as they are accelerated and injected by the explosive magnetic reconfiguration that occurs during substorms.

\section{Plasma Waves}

Additionally, plasma waves, which are electric and magnetic field vibrations that interact with the charged particles, play an especially important role in modifying distribution of radiation belt electrons on timescales of a few hours to days. This complicates analyses of radiation belt electrons. Figure 3 (adapted from Summers et al. [1998]) is a sketch of the regions where the relevant plasma waves occur relative to radiation belt electron drift paths.

One important group of waves is the large-scale oscillations in the ultra-lowfrequency (ULF) range. With periods of around one to tens of minutes, ULF waves can have a resonant interaction with the drift motion of relativistic electrons, spatially diffusing these particles. The resulting radial diffusion either brings plasma sheet electrons into the outer zone belt, or spreads locally accelerated electron peaks outward. This diffusion is typically a relatively slow (timescales of days) but major process for relativistic electrons, though during strong storms the diffusion timescale can be hours.

Relativistic electrons can also resonate with higher-frequency ULF waves in the period range of 1-10 seconds. The largest source of these oscillations is in the region of ring current-plasmasphere overlap. Here the conditions are right for electromagnetic ion cyclotron (EMIC) waves to be strongly enhanced. These EMIC waves can alter a radiation belt electron's direction of motion, expanding the bounce path until it reaches the upper atmosphere. This results in a large but localized loss of particles.

Waves in the extremely low frequency (ELF; 3 hertz to 3 kilohertz) and very low frequency (VLF; 3-30 kilohertz) ranges can also resonate with the relativistic electrons. These waves can have several effects, depending on the location and characteristics of the waves. VLF "chorus" waves, so named because of their characteristic "chirp" pattern, are created in the inner magnetosphere by freshly injected plasma sheet electrons, and therefore exist outside the plasmasphere across the entire dawnside of the inner magnetosphere. On the nightside of dawn, they are typically confined to near-equatorial latitudes, and their resonant interaction with relativistic electrons is primarily manifested as an energy diffusion process. This process results in a net 
acceleration of the radiation belt electrons as they drift through this region, and is consid-

ered a major candidate for local acceleration of relativistic electrons in the outer zone.

On the daytime side of dawn, chorus waves typically occur away from the magnetic equator, and the resonant interaction with the relativistic electrons is dominated by diffusion in the direction of motion. As with EMIC waves, this is a loss process, resulting in electrons being dumped into the upper atmosphere. So the same type of plasma wave can be a source or loss of relativistic electrons, depending on where and when the electrons interact with the particles.

ELF and VLF plasma waves also exist inside the plasmasphere, in the form of plasmaspheric hiss, lightning-generated whistler waves, and noise from ground-based VLFfrequency transmitters. The dominant interaction with relativistic electrons for all of these plasma waves is directional diffusion, and they therefore cause a loss of the radiation belts inside the plasmasphere. These waves are responsible for the creation of the electron slot region (see Figure 1) between the inner and outer radiation belts, and they can clear the outer plasmaspheric region of relativistic electrons in a matter of days to weeks.

\section{An Integrated View of Radiation Belt Enhancements}

How do all of these processes relate to each other and add up to radiation belt enhancements or depletions? We don't know yet, and this is an active research area. As indicated in Figure 3, relativistic electron dynamics strongly depend on the relative positions of the plasmasphere, ring current, and radiation belts, and the occurrence of the various waves with which they interact.

Note that the ultimate drivers in the solar wind often produce competing effects. For instance, radiation belt electron enhancements occur when radial diffusion is enhanced and when the plasmasphere is eroded, which allows nightside chorus waves to exist well within the inner magnetosphere. Both of these effects occur when magnetospheric convection is enhanced. On the other hand, enhanced convection also builds up the ring current, which inflates the magnetic field, causing the Dst effect and increasing magnetopause shadowing. The storm-time ring current also excites EMIC waves, which deplete the radiation belts. This competition between simultaneous source and loss mechanisms is why some geomagnetic storms enhance the radiation belts while others do not [Reeves, 1998]. To further complicate the issue, the fast drift periods of radiation belt particles mean they are influenced by the entire magnetic configuration of the belts and by the mix of plasma waves that the particle encounters around its drift path.

Fortunately, multispacecraft measurements and increasingly more sophisticated theory and modeling show great promise in ultimately revealing the most important processes and in providing improved physical models with predictive capability. Data assimilation is also expected to play a key role in refining and improving model results. Future missions include the NASA
Living With a Star Radiation Belt Storm Probes (LWS RBSP) and the Canadian Outer Radiation Belt Injection, Transport, Acceleration and Loss Satellite (ORBITALS) missions. In addition, the NASA LWS Targeted Research and Technology program occasionally organizes teams of researchers to examine the problem of radiation belt enhancements.

\section{Acknowledgments}

The authors would like to thank the NASA Living With a Star Targeted Research and Technology program for supporting research of the physical processes responsible for radiation belt enhancements.

\section{References}

Kim, H.-J., and A.A.Chan (1997), Fully adiabatic changes in storm time relativistic electron fluxes, J. Geophys. Res., 102,22,107.

Reeves, G. D. (1998), Relativistic electrons and magnetic storms: 1992-1995, Geophys. Res. Lett., 25 , 3265 .

Roederer, J. G. (1970), Dynamics of Geomagnetically Trapped Radiation, vol. 2, Springer, New York.

Summers, D., R. M. Thorne, and FXiao (1998), Relativistic theory of wave-particle resonant diffusion with application to electron acceleration in the magnetosphere, J. Geophys. Res., 103, 20,487.

\section{Author Information}

Michael W. Liemohn, Atmospheric, Oceanic, and Space Sciences Department, University of Michigan, Ann Arbor; E-mail: liemohn@umich.edu; Anthony A. Chan, Physics and Astronomy Department, Rice University, Houston, Tex. 
Page 425

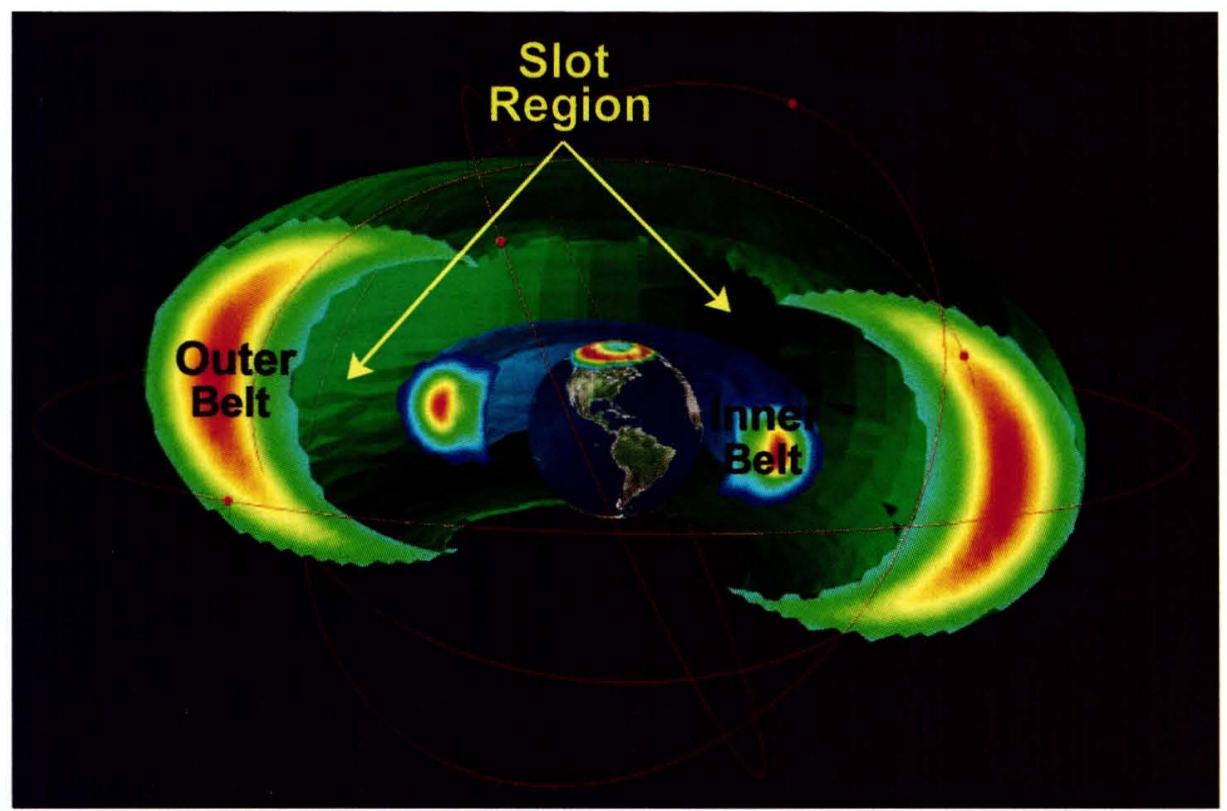

Fig. 1. Energetic electron flux, showing the inner and outer radiation belts. Warm colors indicate high flux intensity, while cool colors indicate low flux intensity values. Also shown are the orbits of three Global Positioning System (GPS) spacecraft and one geosynchronous spacecraft (the equatorial orbit). Image from AF-GEOSpace.

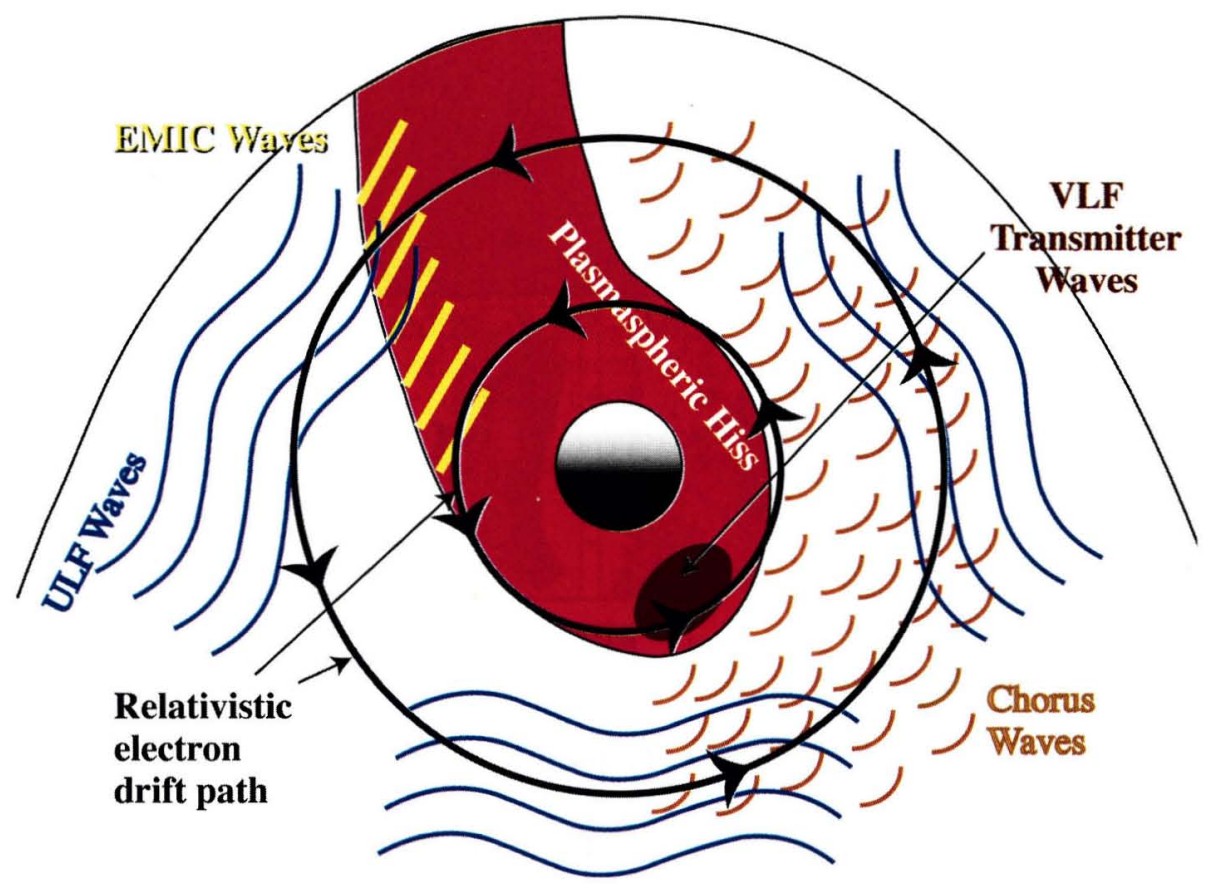

Fig. 3. A schematic of how relativistic electron paths pass through various plasma wave regions over the course of a drift period. The inner drift path will experience mostly loss processes, while the outer drift path encounters a mixture of acceleration and loss processes. Adapted from Summers et al. [1998]. 